\title{
Application of the double Laplace Adomian decomposition method for solving linear singular one dimensional thermo-elasticity coupled system
}

\author{
Hassan Eltayeb ${ }^{\mathrm{a}}$, Adem Kılıçman ${ }^{\mathrm{b}, *}$, Said Mesloub ${ }^{\mathrm{a}}$ \\ ${ }^{a}$ Mathematics Department, College of Science, King Saud University, P. O. Box 2455, Riyadh 11451, Saudi Arabia. \\ ${ }^{b}$ Department of Mathematics, University Putra Malaysia, 43400 Serdang, Selangor, Malaysia. \\ Communicated by I. Argyros
}

\begin{abstract}
In the present work, the Adomain decomposition and double Laplace transform methods are combined to solve linear singular one dimensional hyperbolic equation and linear singular one dimensional thermo-elasticity coupled system. Also we address the convergence of double Laplace transform decomposition method. Moreover, some examples are given to establish our method. (C)2017 All rights reserved.
\end{abstract}

Keywords: Double Laplace transform, inverse Laplace transform, nonlinear hyperbolic equation, single Laplace transform, decomposition methods, thermo-elasticity. 2010 MSC: 35A44, 65M55.

\section{Introduction}

In general, it is reported in the literature that finding the exact solutions for partial differential equations is a complicated task. Therefore, some recent approximate methods to overcome this task have been improved, such as homotopy perturbation method [4, 19], combined Laplace transforms and decomposition method [6] to solve first order differential equation, an auxiliary parameter method using Adomain polynomials and Laplace transformation have been powerfully combined [13] to study the nonlinear differential equation. The one dimensional nonlinear hyperbolic equation with Bessel operator is one of the fundamental nonlinear wave equations having many applications in science. The energy-integral method is used to handle nonlinear singular one dimensional hyperbolic equation [7]. In [18] authors studied a nonlocal mixed problem for a nonlinear singular system of thermo-elasticity by using a functional analysis approach and an iteration method. The goal of this paper is to study the application of the modified double Laplace transform decomposition method to solve a linear singular one dimensional hyperbolic equation and linear singular one dimensional thermo-elasticity coupled system. The convergence of Adomian's method has been studied by several authors $[1-3,5,10]$. Now, we give the following definitions

\footnotetext{
*Corresponding author

Email addresses: hgadain@ksu.edu.sa (Hassan Eltayeb), akilic@upm.edu .my (Adem Kılıçman), mesloub@ksu.edu.sa (Said Mesloub)

doi:10.22436/jnsa.010.01.26
}

Received 2016-10-10 
which are given by $[8,9,14,15]$. The double Laplace transform is defined as

$$
\mathrm{L}_{x} \mathrm{~L}_{\mathrm{t}}[\mathrm{f}(\mathrm{x}, \mathrm{s})]=\mathrm{F}(\mathrm{q}, \mathrm{s})=\int_{0}^{\infty} e^{-q x} \int_{0}^{\infty} e^{-s t} f(x, \mathrm{t}) \mathrm{dt} \mathrm{dx},
$$

where $x, t>0$ and $q, s$ are complex values, and further double Laplace transform of the first order partial derivatives is given by

$$
\mathrm{L}_{x} \mathrm{~L}_{\mathrm{t}}\left[\frac{\partial v(x, \mathrm{t})}{\partial x}\right]=\mathrm{qV}(\mathrm{q}, \mathrm{s})-\mathrm{V}(0, \mathrm{~s})
$$

Similarly the double Laplace transform for second order derivative with respect to the variables $x$ and $t$ are defined as follows

$$
\begin{aligned}
& L_{x} L_{t}\left[\frac{\partial^{2} u(x, t)}{\partial^{2} \chi}\right]=q^{2} u(q, s)-q u(0, s)-\frac{\partial u(0, s)}{\partial x}, \\
& L_{x} L_{t}\left[\frac{\partial^{2} u(x, t)}{\partial^{2} t}\right]=s^{2} u(q, s)-s u(q, 0)-\frac{\partial u(q, 0)}{\partial t} .
\end{aligned}
$$

First of all we need the following lemma for future use in this paper.

Lemma 1.1. Double Laplace transform of the non-constant coefficient second order partial derivative $x^{n} \frac{\partial^{2} u}{\partial t^{2}}$ and the function $x^{n} f(x, t)$ given by

$$
(-1)^{n} \frac{d^{n}}{d q^{n}}\left[L_{x} L_{t}\left(\frac{\partial^{2} u}{\partial t^{2}}\right)\right]=L_{x} L_{t}\left(x^{n} \frac{\partial^{2} u}{\partial t^{2}}\right)
$$

and

$$
L_{x} L_{t}\left(x^{n} f(x, t)\right)=(-1)^{n} \frac{d^{n}}{d q^{n}}\left[L_{x} L_{t}(f(x, t))\right]=(-1)^{n} \frac{d^{n} F(q, s)}{d q^{n}} .
$$

We can prove this lemma by the aid of the definition of double Laplace transform in (1.1), (1.3) and (1.2).

\section{Singular one dimensional hyperbolic equation}

In this part of the paper we discuss how to obtain the solution of the singular one dimensional hyperbolic equation:

$$
\frac{\partial^{2} u}{\partial t^{2}}=\frac{1}{x}\left(x \frac{\partial u}{\partial x}\right)_{x}+f(x, t)
$$

subject to

$$
u(x, 0)=f_{1}(x), \quad \frac{\partial u(x, 0)}{\partial t}=f_{2}(x)
$$

where $\frac{1}{x}\left(x \frac{\partial u}{\partial x}\right)_{x}$ is called Bessel operator and $f(x, t), f_{1}(x)$ and $f_{2}(x)$ are known functions. In the following theorem we apply modified double Laplace decomposition methods.

Theorem 2.1. The solution of the singular one dimensional hyperbolic equation given in (2.1) exists and is given by

$$
\begin{aligned}
u(x, t)= & f_{1}(x)+t f_{2}(x)-L_{q}^{-1} L_{s}^{-1}\left[\frac{1}{s^{2}} L_{x} L_{t}\left[\int_{0}^{q} x f(x, t) d q\right]\right] \\
& -L_{q}^{-1} L_{s}^{-1}\left[\frac{1}{s^{2}} L_{x} L_{t}\left[\int_{0}^{q}\left(x \frac{\partial}{\partial x} \sum_{n=0}^{\infty} u_{n}\right)_{x} d q\right]\right]
\end{aligned}
$$


where

$$
u(x, t)=\sum_{n=0}^{\infty} u_{n}(x, t),
$$

and $\mathrm{L}_{\chi} \mathrm{L}_{\mathrm{t}}$ double Laplace transform with respect to $\mathrm{x}, \mathrm{t}$ and $\mathrm{L}_{\mathrm{q}}^{-1} \mathrm{~L}_{\mathrm{s}}^{-1}$ double inverse Laplace transform with respect to $\mathrm{q}, \mathrm{s}$, further $\mathrm{A}_{\mathrm{n}}$ represents the linear terms. Here we provided double inverse Laplace transform with respect to $\mathrm{p}$ and $\mathrm{s}$ exists for each terms in the right hand side of (2.3).

Proof. We first apply the double Laplace transform to equation in (2.1), then in presence of initial conditions (2.2), and the differentiation property of double Laplace transform together with Lemma 1.1, we obtain:

$$
\begin{aligned}
\frac{d U(q, s)}{d p}= & \frac{1}{s} \frac{d F_{1}(q)}{d q}+\frac{1}{s^{2}} \frac{d F_{2}(q)}{d q} \\
& -\frac{1}{s^{2}} L_{x} L_{t}\left[\left(x \frac{\partial u}{\partial x}\right)_{x}\right]-\frac{1}{s^{2}} L_{x} L_{t}[x f(x, t)] .
\end{aligned}
$$

By applying the integral for both sides of (2.4) from 0 to $q$, we get

$$
\begin{aligned}
u(q, s)= & \frac{F_{1}(q)}{s}+\frac{F_{2}(q)}{s^{2}}-\frac{1}{s^{2}} \int_{0}^{q} L_{x} L_{t}[x f(x, t)] d q \\
& -\frac{1}{s^{2}} \int_{0}^{q} L_{x} L_{t}\left[\left(x \frac{\partial u}{\partial x}\right)_{x}\right] d q .
\end{aligned}
$$

The double Laplace Adomain decomposition methods (DLADM) defines the solution of linear singular one dimensional hyperbolic equation as $u(x, t)$ by the infinite series

$$
u(x, t)=\sum_{n=0}^{\infty} u_{n}(x, t)
$$

By applying double inverse Laplace transform for (2.5) we obtain

$$
\begin{aligned}
u(x, t)= & f_{1}(x)+t f_{2}(x) \\
& -L_{q}^{-1} L_{s}^{-1}\left[\frac{1}{s^{2}} \int_{0}^{q} L_{x} L_{t}[x f(x, t)] d q\right] \\
& -L_{q}^{-1} L_{s}^{-1}\left[\frac{1}{s^{2}} \int_{0}^{q} L_{x} L_{t}\left[\left(x \frac{\partial u}{\partial x}\right)_{x}\right] d q\right],
\end{aligned}
$$

then the general decomposition formula for (2.6) is given by

$$
\begin{aligned}
u(x, t)= & f_{1}(x)+t f_{2}(x)-L_{q}^{-1} L_{s}^{-1}\left[\frac{1}{s^{2}} L_{x} L_{t}\left[\int_{0}^{q} x f(x, t) d q\right]\right] \\
& -L_{q}^{-1} L_{s}^{-1}\left[\frac{1}{s^{2}} L_{x} L_{t}\left[\int_{0}^{q}\left(x \frac{\partial}{\partial x} \sum_{n=0}^{\infty} u_{n}\right)_{x} d q\right]\right] .
\end{aligned}
$$

In particular, we have

$$
u_{0}(x, t)=f_{1}(x)+t f_{2}(x)-L_{q}^{-1} L_{s}^{-1}\left[\frac{1}{s^{2}} L_{x} L_{t}\left[\int_{0}^{q}(x f(x, t)) d q\right]\right]
$$

and

$$
u_{n+1}(x, t)=-L_{q}^{-1} L_{s}^{-1}\left[\frac{1}{s^{2}} L_{x} L_{t}\left[\int_{0}^{q}\left(x \frac{\partial}{\partial x} \sum_{n=0}^{\infty} u_{n}\right)_{x} d q\right]\right],
$$


where $n=0,1,2, \cdots$, and by calculating the terms $u_{0}, u_{1}, u_{2}, \cdots$ we obtain the solution as follows

$$
u(x, t)=u_{0}+u_{1}+u_{2}+\cdots .
$$

In order to establish our method for solving the singular one-dimensional hyperbolic equation, we consider the following example.

Example 2.2. Let us consider the one dimensional hyperbolic equation

$$
\frac{\partial^{2} u}{\partial t^{2}}-\frac{1}{x}\left(x \frac{\partial u}{\partial x}\right)_{x}=-x^{2} \sin t-4 \sin t
$$

associated with the initial conditions

$$
u(x, 0)=0, \quad \frac{\partial u(x, 0)}{\partial t}=x^{2} .
$$

By taking the double and single Laplace transform for (2.7) and (2.8) respectively, we obtain

$$
\frac{d u(q, s)}{d q}=-\frac{6}{q^{4} s^{2}}+\frac{6}{q^{4} s^{2}\left(s^{2}+1\right)}+\frac{4}{q^{2} s^{2}\left(s^{2}+1\right)}-\frac{1}{s^{2}} L_{x} L_{t}\left[\left(x \frac{\partial u}{\partial x}\right)_{x}\right] .
$$

By taking integral in both sides of (2.9) from 0 to $q$, we have

$$
u(q, s)=\frac{2}{q^{3} s^{2}}-\frac{2}{q^{3} s^{2}\left(s^{2}+1\right)}-\frac{4}{q s^{2}\left(s^{2}+1\right)}-\frac{1}{s^{2}} \int_{0}^{q} L_{x} L_{t}\left[\left(x \frac{\partial u}{\partial x}\right)_{x}\right] d q .
$$

By using double inverse Laplace transform, we have

$$
u(x, t)=x^{2} \sin t+4 \sin t-4 t-L_{q}^{-1} L_{s}^{-1}\left(\frac{1}{s^{2}} \int L_{x} L_{t}\left[\left(x \frac{\partial u}{\partial x}\right)_{x}\right] d q\right),
$$

and by using equation (2.3), we obtain

$$
\begin{gathered}
\sum_{n=0}^{\infty} u_{n}(x, t)=x^{2} \sin t+4 \sin t-4 t-L_{q}^{-1} L_{s}^{-1}\left(\frac{1}{s^{2}} \int_{0}^{q} L_{x} L_{t}\left[\left(x \frac{\partial}{\partial x} \sum_{n=0}^{\infty} u_{n}\right){ }_{x}\right] d q\right), \\
u_{0}=x^{2} \sin t+4 \sin t-4 t .
\end{gathered}
$$

The other components are given by

$$
u_{n+1}=-L_{q}^{-1} L_{s}^{-1}\left(\frac{1}{s^{2}} \int_{0}^{q} L_{x} L_{t}\left[\left(x \frac{\partial}{\partial x} \sum_{n=0}^{\infty} u_{n}\right)_{x}\right] d q\right) .
$$

Therefore

$$
\begin{aligned}
& \mathrm{u}_{1}=-\mathrm{L}_{\mathrm{q}}^{-1} \mathrm{~L}_{\mathrm{s}}^{-1}\left(\frac{1}{\mathrm{~s}^{2}} \int_{0}^{\mathrm{q}} \mathrm{L}_{\mathrm{x}} \mathrm{L}_{\mathrm{t}}\left[\left(x \frac{\partial}{\partial x} \mathrm{u}_{0}\right)_{\chi}\right] \mathrm{dq}\right), \\
& \mathrm{u}_{1}=4 \mathrm{t}-4 \sin \mathrm{t},
\end{aligned}
$$

and

$$
u_{2}=0 \text {. }
$$

It is obvious that the rest coming terms all zeros, we have

$$
u(x, t)=u_{0}+u_{1}+\cdots .
$$

Therefore, the exact solution is given by

$$
u(x, t)=x^{2} \sin t
$$




\section{Linear singular one dimensional thermo-elasticity coupled system}

In this section of the paper, we apply our technique to solve the linear singular one dimensional thermo-elasticity coupled system given below

$$
\begin{aligned}
& \frac{\partial^{2} u}{\partial t^{2}}-\frac{1}{x^{n}}\left(x^{n} \frac{\partial u}{\partial x}\right)_{x}+x \frac{\partial v}{\partial x}=f(x, t), \quad x \in \Omega, \\
& \frac{\partial v}{\partial t}-\frac{1}{x^{n}}\left(x^{n} \frac{\partial v}{\partial x}\right)_{x}+x \frac{\partial^{2} u}{\partial x \partial t}=g(x, t), \quad t>0,
\end{aligned}
$$

subject to

$$
u(x, 0)=f_{1}(x), \quad \frac{\partial u(x, 0)}{\partial t}=f_{2}(x), \quad v(x, 0)=g_{1}(x),
$$

where $\frac{1}{x^{n}}\left(x^{n} \frac{\partial u}{\partial x}\right)_{x}$ and $\frac{1}{x^{n}}\left(x^{n} \frac{\partial v}{\partial x}\right)_{x}$ are called Bessel's operators, $f(x, t), g(x, t), f_{1}(x), f_{2}(x)$ and $g_{1}(x)$ are known functions and $n=1,2,3, \cdots$. To obtain the solution of Linear singular one dimensional thermoelasticity coupled system of (3.1), we apply our method as follows. On using the definition of partial derivatives of the double Laplace transform and single Laplace transform for (3.1) and (3.2) respectively and Lemma 1.1, we get

$$
\begin{aligned}
\frac{d^{n} U(q, s)}{d q^{n}}= & \frac{d^{n} F_{1}(q)}{s d q^{n}}+\frac{d^{n} F_{2}(q)}{s^{2} d q^{n}}+\frac{d^{n} F(q, s)}{s^{2} d q^{n}} \\
& +\frac{(-1)^{n}}{s^{2}} L_{x} L_{t}\left[\left(x^{n} \frac{\partial u}{\partial x}\right)_{x}-x^{n+1} \frac{\partial v}{\partial x}\right],
\end{aligned}
$$

and

$$
\begin{aligned}
\frac{d^{n} V(q, s)}{d q^{n}}= & \frac{d^{n} G_{1}(q)}{s d q^{n}}+\frac{d^{n} G(q, s)}{s d q^{n}} \\
& +\frac{(-1)^{n}}{s} L_{x} L_{t}\left[\left(x^{n} \frac{\partial v}{\partial x}\right)_{x}-x^{n+1} \frac{\partial^{2} u}{\partial x \partial t}\right],
\end{aligned}
$$

where $F(q, s), G(q, s), F_{1}(q), F_{2}(q)$ and $G_{1}(q)$ are double and single Laplace transforms of $f(x, t)$, $g(x, t), f_{1}(x), f_{2}(x)$ and $g_{1}(x)$ respectively. By integrating $n$ times for both sides of (2.4) from 0 to $q$ with respect to $q$, we obtain

$$
\begin{aligned}
\mathrm{u}(\mathrm{q}, \mathrm{s})= & \iint \ldots \int\left(\frac{\mathrm{d}^{n} F_{1}(\mathrm{q})}{s d q^{n}}+\frac{d^{n} F_{2}(q)}{s^{2} d q^{n}}+\frac{d^{n} F(q, s)}{s^{2} d q^{n}}\right) d q \ldots d q d q \\
& \times \frac{(-1)^{n}}{s^{2}} \iint \ldots \int\left(L_{x} L_{t}\left[\left(x^{n} \frac{\partial u}{\partial x}\right)_{x}-x^{n+1} \frac{\partial v}{\partial x}\right]\right) d q \ldots d q d q
\end{aligned}
$$

and

$$
\begin{aligned}
V(q, s)= & \iint \ldots \int\left(\frac{d^{n} G_{1}(q)}{s d q^{n}}+\frac{d^{n} G(q, s)}{s d q^{n}}\right) d q \ldots d q d q \\
& +\frac{(-1)^{n}}{s} \iint \ldots \int L_{x} L_{t}\left[\left(x^{n} \frac{\partial v}{\partial x}\right)_{x}-x^{n+1} \frac{\partial^{2} u}{\partial x \partial t}\right] d q \ldots d q d q .
\end{aligned}
$$

The double Laplace Adomain decomposition methods (DLADM) defines the solution of the system as $u(x, t)$ and $v(x, t)$ by the infinite series

$$
u(x, t)=\sum_{n=0}^{\infty} u_{n}(x, t), \quad v(x, t)=\sum_{n=0}^{\infty} v_{n}(x, t) .
$$


By applying double inverse Laplace transform for (3.3) and (3.4) and using (3.5), we have

$$
\begin{aligned}
u(x, t)= & L_{q}^{-1} L_{s}^{-1}\left[\iint \ldots \int\left(\frac{d^{n} F_{1}(q)}{s d q^{n}}+\frac{d^{n} F_{2}(q)}{s^{2} d q^{n}}+\frac{d^{n} F(q, s)}{s^{2} d q^{n}}\right) d q \ldots d q d q\right] \\
& +L_{q}^{-1} L_{s}^{-1}\left[\frac{(-1)^{n}}{s^{2}} \iint \ldots \int\left(L_{x} L_{t}\left[\left(x^{n} \frac{\partial u}{\partial x}\right)_{x}\right]\right) d q \ldots d q d q\right] \\
& +L_{q}^{-1} L_{s}^{-1}\left[\frac{(-1)^{n+1}}{s^{2}} \iint \ldots \int\left(L_{x} L_{t}\left[x^{n+1} \frac{\partial v}{\partial x}\right]\right) d q \ldots d q\right], \\
v(x, t)= & L_{q}^{-1} L_{s}^{-1}\left[\iint \ldots \int\left(\frac{d^{n} G_{1}(q)}{s d p^{n}}+\frac{d^{n} G(q, s)}{s d p^{n}}\right) d q \ldots d q d q\right] \\
& +L_{q}^{-1} L_{s}^{-1}\left[\frac{(-1)^{n}}{s} \iint \ldots \int\left(L_{x} L_{t}\left[\left(x^{n} \frac{\partial v}{\partial x}\right)\right]\right) d q \ldots d q d q\right] \\
& +L_{q}^{-1} L_{s}^{-1}\left[\frac{(-1)^{n+1}}{s} \iint \ldots \int\left(L_{x} L_{t}\left[x^{n+1} \frac{\partial^{2} u}{\partial x \partial t}\right]\right) d q \ldots d q d q\right] .
\end{aligned}
$$

In particular, if $n=1$, (3.6) and (3.7) becomes

$$
\begin{aligned}
u(x, t)= & f_{1}(x)+t f_{2}(x)+L_{q}^{-1} L_{s}^{-1}\left[\frac{1}{s^{2}} \int_{0}^{q} d F(q, s)\right] \\
& -L_{q}^{-1} L_{s}^{-1}\left[\frac{1}{s^{2}} \int_{0}^{q} L_{x} L_{t}\left[\left(x \frac{\partial u}{\partial x}\right)_{x}-x^{2} \frac{\partial v}{\partial x}\right] d q\right],
\end{aligned}
$$

and

$$
\begin{aligned}
v(x, t)= & g_{1}(x)+L_{q}^{-1} L_{s}^{-1}\left[\frac{1}{s} \int_{0}^{q} d G(q, s)\right] \\
& -L_{q}^{-1} L_{s}^{-1}\left[\frac{1}{s} \int_{0}^{q}\left(L_{x} L_{t}\left[\left(x \frac{\partial v}{\partial x}\right)_{x}-x^{2} \frac{\partial^{2} u}{\partial x \partial t}\right]\right) d q\right] .
\end{aligned}
$$

By using (3.5) into (3.8) and (3.9) we get

$$
\begin{aligned}
\sum_{n=0}^{\infty} u_{n}(x, t)= & f_{1}(x)+t_{2}(x)+L_{q}^{-1} L_{s}^{-1}\left[\frac{1}{s^{2}} \int_{0}^{q} d F(q, s)\right] \\
& -L_{q}^{-1} L_{s}^{-1}\left[\frac{1}{s^{2}} \int_{0}^{q} L_{x} L_{t}\left(x\left(\sum_{n=0}^{\infty} u_{n x}(x, t)\right)\right)_{x} d q\right] \\
& +L_{q}^{-1} L_{s}^{-1}\left[\frac{1}{s^{2}} \int_{0}^{q} L_{x} L_{t}\left[x^{2} \sum_{n=0}^{\infty} v_{n x}(x, t)\right] d q\right]
\end{aligned}
$$

and

$$
\begin{aligned}
\sum_{n=0}^{\infty} v_{n}(x, t)= & g_{1}(x)+L_{q}^{-1} L_{s}^{-1}\left[\frac{1}{s} \int_{0}^{q} d G(q, s)\right] \\
& -L_{q}^{-1} L_{s}^{-1}\left[\frac{1}{s} \int\left(L_{x} L_{t}\left[\left(x \sum_{n=0}^{\infty} v_{n x}(x, t)\right)_{x}\right]\right) d q\right] \\
& +L_{q}^{-1} L_{s}^{-1}\left[\frac{1}{s} \int\left(L_{x} L_{t}\left[x^{2} \frac{\partial^{2}}{\partial x \partial t}\left(\sum_{n=0}^{\infty} u_{n}(x, t)\right)\right]\right) d q\right]
\end{aligned}
$$


In particular

$$
\begin{aligned}
& u_{0}(x, t)=f_{1}(x)+t f_{2}(x)+L_{q}^{-1} L_{s}^{-1}\left[\frac{1}{s^{2}} \int_{0}^{q} d F(q, s)\right], \\
& v_{0}(x, t)=g_{1}(x)+L_{q}^{-1} L_{s}^{-1}\left[\frac{1}{s} \int_{0}^{q} d G(q, s)\right] .
\end{aligned}
$$

Generally we have

$$
\begin{aligned}
u_{n+1}(x, t)= & -L_{q}^{-1} L_{s}^{-1}\left[\frac{1}{s^{2}} \int_{0}^{q} L_{x} L_{t}\left[\left(x \sum_{n=0}^{\infty} u_{n x}(x, t)\right)_{x}\right] d q\right] \\
& +L_{q}^{-1} L_{s}^{-1}\left[\frac{1}{s^{2}} \int_{0}^{q} L_{x} L_{t}\left[x^{2} \sum_{n=0}^{\infty} v_{n x}(x, t)\right] d q\right], \\
v_{n+1}(x, t)= & -L_{q}^{-1} L_{s}^{-1}\left[\frac{1}{s} \int\left(L_{x} L_{t}\left[\left(\sum_{n=0}^{\infty} v_{n x}(x, t)\right)_{x}\right]\right) d q\right] \\
& +L_{q}^{-1} L_{s}^{-1}\left[\frac{1}{s} \int\left(L_{x} L_{t}\left[x^{2} \frac{\partial^{2}}{\partial x \partial t}\left(\sum_{n=0}^{\infty} u_{n}(x, t)\right)\right]\right) d q\right] .
\end{aligned}
$$

By calculate the terms $u_{0}, u_{1}, \cdots$ and $v_{0}, v_{1}, \cdots$, we get the solution of our system as

$$
u(x, t)=u_{0}+u_{1}+\cdots, \text { and } v(x, t)=v_{0}+v_{1}+\cdots .
$$

In the following example we consider $n=1$ in (3.1) as:

Example 3.1. Consider the following linear singular one dimensional thermo-elasticity coupled system

$$
\begin{aligned}
& \frac{\partial^{2} u}{\partial t^{2}}-\frac{1}{x}\left(x \frac{\partial u}{\partial x}\right)_{x}+x \frac{\partial v}{\partial x}=-x^{2} \sin t-4 \sin t+2 x^{2} e^{t}, \quad x \in \Omega, \\
& \frac{\partial v}{\partial t}-\frac{1}{x}\left(x \frac{\partial v}{\partial x}\right)_{x}+x \frac{\partial^{2} u}{\partial x \partial t}=x^{2} e^{t}-4 e^{t}+2 x^{2} \cos t, \quad t>0,
\end{aligned}
$$

subject to

$$
u(x, 0)=0, \frac{\partial u(x, 0)}{\partial t}=x^{2}, v(x, 0)=x^{2} .
$$

By using modified double Laplace decomposition methods for (3.13), (3.14) and apply (3.8), (3.9), we have

$$
\begin{aligned}
u(x, t)= & x^{2} \sin t+4 \sin t-4 t+2 x^{2} e^{t}-2 x^{2} t-2 x^{2} \\
& -L_{q}^{-1} L_{s}^{-1}\left[\frac{1}{s^{2}} \int_{0}^{q} L_{x} L_{t}\left[\left(x \frac{\partial u}{\partial x}\right)_{x}-x^{2} \frac{\partial v}{\partial x}\right] d q\right],
\end{aligned}
$$

and

$$
\begin{aligned}
v(x, t)= & x^{2} e^{t}-4 e^{t}+2 x^{2} \sin t-4 \\
& -L_{q}^{-1} L_{s}^{-1}\left[\frac{1}{s} \int\left(L_{x} L_{t}\left[\left(x \frac{\partial v}{\partial x}\right)_{x}-x^{2} \frac{\partial^{2} u}{\partial x \partial t}\right]\right) d q\right] .
\end{aligned}
$$

On using (3.10), (3.11) and applying (3.12), we get

$$
\begin{aligned}
& u_{0}(x, t)=x^{2} \sin t+4 \sin t-4 t+2 x^{2} e^{t}-2 x^{2} t-2 x^{2}, \\
& v_{0}(x, t)=x^{2} e^{t}-4 e^{t}+2 x^{2} \sin t-4,
\end{aligned}
$$




$$
\begin{aligned}
& u_{1}(x, t)=-4 t+4 x^{2} \sin t-4 \sin t+8 e^{t}-8-\frac{4}{3} t^{3}-4 t^{2}-2 x^{2} e^{t}-2 x^{2} t+2 x^{2}, \\
& v_{1}(x, t)=4 x^{2} e^{t}+4 e^{t}-2 x^{2} \sin t+4-8 \cos t-4 x^{2},
\end{aligned}
$$

and

$$
\begin{aligned}
& u_{2}(x, t)=8 t-4 x^{2} \sin t-16 \sin t-8 e^{t}+8+\frac{4}{3} t^{3}+4 t^{2}-4 x^{2} e^{t}+8 x^{2} t+4 x^{2}+2 x^{2} t^{2} \\
& v_{2}(x, t)=4 x^{2} e^{t}+16 e^{t}-8 x^{2} \sin t-24+8 \cos t-4 x^{2}+4 x^{2} t-16 t
\end{aligned}
$$

Therefore, the approximate solution is

$$
u(x, t)=u_{0}+u_{1}+\cdots, \text { and } v(x, t)=v_{0}+v_{1}+\cdots .
$$

We obtain the closed form solution

$$
u(x, t)=x^{2} \sin t, \quad \text { and } \quad v(x, t)=x^{2} e^{t} .
$$

\section{Convergence analysis}

In this section, we discuss the convergence analysis of the modified double Laplace decomposition methods for the nonlinear singular one dimensional hyperbolic equation. We propose to extend this idea given in [16, 17]. First of all let us consider the Hilbert space $H=L_{\mu}^{2}((a, b) \times[0, T])$, where $a \ll 0$ with following scalar product

$$
\begin{aligned}
& u:(a, b) \times[0, T] \rightarrow \mathbb{R}, \text { with }\|u\|_{H}^{2}=\int_{Q} x u^{2}(x, t) d x d t \\
& (u, v)=\int_{Q} x u(x, t) v(x, t) d x d t
\end{aligned}
$$

where $\mathrm{Q}=(\mathrm{a}, \mathrm{b}) \times[0, \mathrm{~T}]$ and

$$
H=\left\{\begin{array}{c}
(u, v):(a, b) \times[0, T], \text { with } \\
L_{p}^{-1} L_{s}^{-1}\left[\frac{1}{s^{2}} \int_{0}^{p} L_{x} L_{t}[u(x, t)](p, s) d p\right](x, t)<\infty
\end{array}\right\} .
$$

Problem: We consider the nonlinear singular one dimensional hyperbolic equation that is given by

$$
\frac{\partial^{2} u}{\partial t^{2}}=\frac{1}{x} \frac{\partial}{\partial x}\left(x \frac{\partial u}{\partial x}\right)+u \frac{\partial u}{\partial x}+f(u), \quad t>0,
$$

for all $u, v \in H$. We define $H$ as $H=L_{\mu}^{2}((a, b) \times[0, T])$ and

$$
\begin{aligned}
& u:(a, b) \times[0, T] \rightarrow \mathbb{R} \times \mathbb{R}, \text { with }\|u\|_{H}^{2}=\int_{Q} x u^{2}(x, t) d x d t \\
& (u, v)=\int_{Q} x u(x, t) v(x, t) d x d t,
\end{aligned}
$$

where $\mathrm{Q}=(\mathrm{a}, \mathrm{b}) \times[0, \mathrm{~T}]$ and

$$
H=\left\{\begin{array}{c}
(u, v):(a, b) \times[0, T], \text { with } \\
L_{p}^{-1} L_{s}^{-1}\left[\frac{1}{s^{2}} \int_{0}^{q} L_{x} L_{t}[u(x, t)](q, s) d q\right](x, t)<\infty
\end{array}\right\} .
$$

Multiplying both sides of (4.1) by $x$ and write the equation in the operator form

$$
L(u)=x \frac{\partial^{2} u}{\partial t^{2}}=\frac{\partial u}{\partial x}+x \frac{\partial^{2} u}{\partial x^{2}}+\frac{1}{2} x \frac{\partial u^{2}}{\partial x}+x f(u), u=u(x, t), t>0,
$$

where $|x| \leqslant b$. Since $L$ is hemicontinuous operator, then we have the following definition. 


\section{Definition 4.1.}

(H1)

$$
\|\mathrm{u}-v\|^{2} \leqslant \frac{1}{\mathrm{k}}(\mathrm{L}(\mathrm{u})-\mathrm{L}(v), \mathrm{u}-v), \mathrm{k}>0, \quad \forall \mathrm{u}, v \in \mathrm{H} .
$$

(H2) For any positive constant $N^{*}$, there exists a constant $C\left(N^{*}\right)>0$ such that for $u, v \in H$ with $N^{*} \geqslant$ $\|\mathrm{u}\|$, and $\mathrm{N}^{*} \geqslant\|v\|$ we have:

$$
\|u-v\|\|w\| \geqslant \frac{1}{\mathrm{C}\left(\mathrm{N}^{*}\right)}(\mathrm{L}(\mathrm{u})-\mathrm{L}(v), w),
$$

for each $w \in \mathrm{H}$. For more details see [16, 17].

Now by using the above definition we have the next theorem and we follow [10-12].

Theorem 4.2 (Sufficient condition of convergence). The modified double Laplace decomposition methods applied to the nonlinear singular one dimensional hyperbolic equation (4.2) with homogenous initial condition, converges to a solution.

Proof. Firstly we try to verify the hypothesis $(\mathrm{H} 1)$ for $\mathrm{L}(\mathrm{u})$ of (4.2). By using the definition of $\mathrm{L}$, we have

$$
L(u)-L(v)=\frac{\partial}{\partial x}(u-v)+x \frac{\partial^{2}}{\partial x^{2}}(u-v)+\frac{1}{2} x \frac{\partial}{\partial x}\left(u^{2}-v^{2}\right)+x(f(u)-f(v)),
$$

therefore,

$$
\begin{aligned}
(L(u)-L(v), u-v)= & \left(\frac{\partial}{\partial x}(u-v), u-v\right) \\
& +\left(x \frac{\partial^{2}}{\partial x^{2}}(u-v), u-v\right) \\
& +\left(\frac{1}{2} x \frac{\partial}{\partial x}\left(u^{2}-v^{2}\right), u-v\right) \\
& +(x(f(u)-f(v)), u-v) .
\end{aligned}
$$

According to the coercive operator the differential operator $\frac{\partial}{\partial x}$ and $\frac{\partial^{2}}{\partial x^{2}}$ in $H$, there exist constants $\alpha, \beta$, $\theta>0$ such that

$$
\left(\frac{\partial}{\partial x}(u-v), u-v\right) \geqslant \alpha\|u-v\|^{2},
$$

and by using Cauchy-Schwarz inequality we have

$$
\begin{aligned}
-\left(x \frac{\partial^{2}}{\partial x^{2}}(u-v), u-v\right) & \leqslant|x|\left\|\frac{\partial^{2}}{\partial x^{2}}(u-v)\right\|\|u-v\| \\
& \leqslant \beta b\|u-v\|^{2} \\
& \Leftrightarrow \\
\left(x \frac{\partial^{2}}{\partial x^{2}}(u-v), u-v\right) & \geqslant-\beta b\|u-v\|^{2},
\end{aligned}
$$

where $\|u\| \leqslant N^{*}$ and $\|v\| \leqslant N^{*}$, and according to the Schwarz inequality, we get

$$
\begin{aligned}
\left(-\frac{1}{2} x \frac{\partial}{\partial x}\left(u^{2}-v^{2}\right), u-v\right) & \leqslant \frac{1}{2}|x|\left\|\frac{\partial}{\partial x}\left(u^{2}-v^{2}\right)\right\|\|u-v\| \\
& \leqslant \frac{1}{2} b \theta\left\|u^{2}-v^{2}\right\|\|u-v\|
\end{aligned}
$$




$$
\begin{aligned}
& \leqslant \frac{1}{2} b \theta\|u+v\|\|u-v\|^{2} \\
& \leqslant b \theta N^{*}\|u-v\|^{2}
\end{aligned}
$$

Hence

$$
\left(\frac{1}{2} x \frac{\partial}{\partial x}\left(u^{2}-v^{2}\right), u-v\right) \geqslant-b \theta N^{*}\|u-v\|^{2} .
$$

By using Schwarz inequality, where $\sigma>0$ as $f$ is Lipschitzian function, we obtain

$$
\begin{aligned}
(-x(f(u)-f(v)), u-v) & \leqslant|x|\|f(u)-f(v)\|\|u-v\| \\
& \leqslant b\|f(u)-f(v)\|\|u-v\| \\
& \leqslant b \sigma\|u-v\|^{2} \\
& \Leftrightarrow \\
(x(f(u)-f(v)), u-v) & \geqslant-b \sigma\|u-v\|^{2} .
\end{aligned}
$$

Substituting (4.4), (4.5), (4.6) and (4.7) into equation (4.3) gives

$$
\begin{aligned}
& (L(u)-L(v), u-v) \geqslant\left(\alpha-\beta b-b \theta N^{*}-b \sigma\right)\|u-v\|^{2}, \\
& (L(u)-L(v), u-v) \geqslant k\|u-v\|^{2} .
\end{aligned}
$$

So the hypothesis (H1) holds, where

$$
k=\alpha-\beta N^{*}-b \theta N^{*}-b \sigma>0 .
$$

Let us now verify hypotheses $(\mathrm{H} 2)$ for $\mathrm{L}(\mathrm{u})$. For any $\mathrm{N}^{*}>0$ there exists a positive constant $\mathrm{C}\left(\mathrm{N}^{*}\right)>0$ such that for all $u, v \in \mathrm{H}$ with $\|u\| \leqslant N^{*},\|v\| \leqslant N^{*}$, and there exist constants $\alpha_{1}, \alpha_{2}, \beta_{1}, \sigma_{1}>0$ such that

$$
(\mathrm{L}(\mathrm{u})-\mathrm{L}(v), w) \leqslant \mathrm{C}\left(\mathrm{N}^{*}\right)\|\mathrm{u}-v\|\|w\|,
$$

for all $w \in \mathrm{H}$. So we have,

$$
\begin{aligned}
(L(u)-L(v), w)= & \left(\frac{\partial}{\partial x}(u-v), w\right) \\
& +\left(x \frac{\partial^{2}}{\partial x^{2}}(u-v), w\right) \\
& +\left(\frac{1}{2} \chi \frac{\partial}{\partial x}\left(u^{2}-v^{2}\right), w\right) \\
& +(x(f(u)-f(v)), w) .
\end{aligned}
$$

The boundedness of the functions $u$ and $v$ and using Schwarz inequality lead to

$$
\begin{aligned}
\left(\frac{\partial}{\partial x}(u-v), w\right) & \leqslant \alpha_{1}\|u-v\|\|w\|, \\
\left(x \frac{\partial^{2}}{\partial x^{2}}(u-v), w\right) & \leqslant b \beta_{1}\|u-v\|\|w\| \\
\left(\frac{1}{2} x \frac{\partial}{\partial x}\left(u^{2}-v^{2}\right), w\right) & \leqslant \frac{1}{2} \alpha_{2}|x|\|u+v\|\|u-v\|\|w\| \\
& \leqslant b \alpha_{2} N^{*}\|u-v\|\|w\|, \\
(x(f(u)-f(v)), w) & \leqslant b \sigma_{1}\|u-v\|\|w\|,
\end{aligned}
$$


so we have

$$
\begin{aligned}
(\mathrm{L}(\mathrm{u})-\mathrm{L}(v), w) & \leqslant\left(\alpha_{1}+\mathrm{b} \beta_{1}+\mathrm{b} \alpha_{2} \mathrm{~N}^{*}+\mathrm{b} \sigma_{1}\right)\|\mathrm{u}-v\|\|w\| \\
& =\mathrm{C}\left(\mathrm{N}^{*}\right)\|\mathrm{u}-v\|\|w\|
\end{aligned}
$$

where

$$
C\left(N^{*}\right)=\left(\alpha_{1}+b \beta_{1}+b \alpha_{2} N^{*}+b \sigma_{1}\right),
$$

and therefore $(\mathrm{H} 2)$ holds. This completes the proof.

Conclusion 4.3. In this work, first, we proposed new modified double Laplace decomposition methods to solve linear singular one dimensional hyperbolic equation and linear singular one dimensional thermo-elasticity coupled system. The efficiency and accuracy of the present scheme are validated through examples. Many classes of single and systems of partial differential equations either linear or nonlinear can be treated and studied by the used method and does not require linearization. Second, we presented a convergence proof of the (DLADM) applied to the nonlinear singular one dimensional hyperbolic equation.

\section{Acknowledgment}

The authors would like to extend their sincere appreciation to the Deanship of Scientific Research at King Saud University for its funding of this research through the Research Group Project number RGP-117.

\section{References}

[1] K. Abbaoui, Y. Cherruault, Convergence of Adomian's method applied to differential equations, Comput. Math. Appl., 28 (1994), 103-109. 1

[2] K. Abbaoui, Y. Cherruault, Convergence of Adomian's method applied to nonlinear equations, Math. Comput. Modelling, 20 (1994), 69-73.

[3] K. Abbaoui, Y. Cherruault, V. Seng, Practical formulae for the calculus of multivariable Adomian polynomials, Math. Comput. Modelling, 22 (1995), 89-93. 1

[4] S. Abbasbandy, Iterated He's homotopy perturbation method for quadratic Riccati differential equation, Appl. Math. Comput., 175 (2006), 581-589. 1

[5] A. Atangana, S. C. Oukouomi Noutchie, On multi-Laplace transform for solving nonlinear partial differential equations with mixed derivatives, Math. Probl. Eng., 2014 (2014), 9 pages. 1

[6] E. Babolian, J. Biazar, A. R. Vahidi, A new computational method for Laplace transforms by decomposition method, Appl. Math. Comput., 150 (2004), 841-846. 1

[7] A. Bouziani, On initial boundary value problem with Dirichlet integral conditions for a hyperbolic equation with the Bessel operator, J. Appl. Math., 2003 (2003), 487-502. 1

[8] C. A. de Moura, A linear uncoupling numerical scheme for the nonlinear coupled thermoelastodynamics equations, Numerical methods, Caracas, (1982), 204-211, Lecture Notes in Math., Springer, Berlin-New York, (1983). 1

[9] H. Eltayeb, A. K1lıcman, A note on solutions of wave, Laplace's and heat equations with convolution terms by using a double Laplace transform, Appl. Math. Lett., 21 (2008), 1324-1329. 1

[10] I. Hashim, M. S. M. Noorani, M. R. Said Al-Hadidi, Solving the generalized Burgers-Huxley equation using the Adomian decomposition method, Math. Comput. Modelling, 43 (2006), 1404-1411. 1, 4

[11] D. Kaya, I. E. Inan, A convergence analysis of the ADM and an application, Appl. Math. Comput., 161 (2005), 10151025.

[12] D. Kaya, I. E. Inan, A numerical application of the decomposition method for the combined KdV-MKdV equation, Appl. Math. Comput., 168 (2005), 915-926. 4

[13] Y. Khan, H. Vázquez-Leal, N. Faraz, An auxiliary parameter method using Adomian polynomials and Laplace transformation for nonlinear differential equations, Appl. Math. Model., 37 (2013), 2702-2708. 1

[14] A. Kılıçman, H. Eltayeb, A note on defining singular integral as distribution and partial differential equations with convolution term, Math. Comput. Modelling, 49 (2009), 327-336. 1

[15] A. Kılıçman, H. E. Gadain, On the applications of Laplace and Sumudu transforms, J. Franklin Inst., 347 (2010), 848 862. 1

[16] T. Mavoungou, Y. Cherruault, Convergence of Adomian's method and applications to non-linear partial differential equations, Kybernetes, 21 (1992), 13-25. 4, 4.1 
[17] T. Mavoungou, Y. Cherruault, Numerical study of Fisher's equation by Adomian's method, Math. Comput. Modelling, 19 (1994), 89-95. 4, 4.1

[18] S. Meslouba, F. Mesloub, On a coupled nonlinear singular thermoelastic system, Nonlinear Anal., 73 (2010), 3195-3208. 1

[19] A. Sadighi, D. D. Ganji, Solution of the generalized nonlinear Boussinesq equation using homotopy perturbation and variational iteration methods, Int. J. Nonlinear Sci. Numer. Simul., 8 (2007), 435-443. 1 